\title{
High Contagiousness and Rapid Spread of Severe Acute Respiratory Syndrome Coronavirus 2
}

\author{
Steven Sanche ${ }^{1}$, Yen Ting Lin ${ }^{1}$, Chonggang Xu, Ethan Romero-Severson, Nick Hengartner, Ruian Ke
}

Severe acute respiratory syndrome coronavirus 2 is the causative agent of the ongoing coronavirus disease pandemic. Initial estimates of the early dynamics of the outbreak in Wuhan, China, suggested a doubling time of the number of infected persons of 6-7 days and a basic reproductive number $\left(\mathrm{R}_{0}\right)$ of $2.2-2.7$. We collected extensive individual case reports across China and estimated key epidemiologic parameters, including the incubation period (4.2 days). We then designed 2 mathematical modeling approaches to infer the outbreak dynamics in Wuhan by using high-resolution domestic travel and infection data. Results show that the doubling time early in the epidemic in Wuhan was 2.3-3.3 days. Assuming a serial interval of 6-9 days, we calculated a median $R_{0}$ value of 5.7 (95\% Cl 3.8-8.9). We further show that active surveillance, contact tracing, quarantine, and early strong social distancing efforts are needed to stop transmission of the virus.

Cevere acute respiratory syndrome coronavirus $\checkmark 2$ (SARS-CoV-2) is the etiologic agent of the current rapidly growing outbreak of coronavirus disease (COVID-19), originating from the city of Wuhan, $\mathrm{Hu}-$ bei Province, China (1). Initially, 41 cases of "pneumonia of unknown etiology" were reported to the World Health Organization by the Wuhan Municipal Health Committee at the end of December 2019 (2). On January 8, 2020, the pathogen was identified (1), and human-to-human transmission was reported soon after. By January 21, most provinces of China had reported COVID-19 cases. By March 16, the outbreak had led to $>170,000$ total confirmed cases and $>6,500$ deaths globally. In a period of 3 months, an outbreak of apparent idiopathic pneumonia had become the COVID-19 pandemic.

Studying dynamics of a newly emerged and rapidly growing infectious disease outbreak, such as COVID-19, is important but challenging because

Author affiliation: Los Alamos National Laboratory, Los Alamos, New Mexico, USA

DOI: https://doi.org/10.3201/eid2607.200282 of the limited amount of data available. In addition, unavailability of diagnostic reagents early in the outbreak, changes in surveillance intensity and case definitions, and overwhelmed healthcare systems confound estimates of the growth of the outbreak based on data. Initial estimates of the exponential growth rate of the outbreak were $0.1-0.14 /$ day (a doubling time of 6-7 days), and a basic reproductive number $\left(\mathrm{R}_{0}\right.$; defined as the average number of secondary cases attributable to infection by an index case after that case is introduced into a susceptible population) ranged from 2.2 to $2.7(1,3-5)$. These estimates were based on 2 broad strategies. First, Li et al. used very early case count data in Wuhan before January 4 (1). However, case count data can be confounded by reservoir spillover events, stochasticities in the initial phase of the outbreak, and low surveillance intensity. The epidemic curve based on symptom onset after January 4 showed a very different growth rate (6). Second, inference was performed by using international flight data and infected persons reported outside of China (3-5). Because of the low numbers of persons traveling abroad compared with the total population size in Wuhan, this approach leads to substantial uncertainties $(7,8)$. Inferences based on a low number of observations are prone to measurement error when data are incomplete or model assumptions are not fully justified; both conditions are common challenges associated with rapid and early outbreak analyses of a new pathogen.

We collected an expanded set of case reports across China on the basis of publicly available information, estimated key epidemiologic parameters, and provided a new estimate of the early epidemic growth rate and $R_{0}$ in Wuhan. Our approaches are based on integration of high-resolution domestic travel data and early infection data reported in provinces other than Hubei to infer outbreak dynamics in

${ }^{1}$ These first authors contributed equally to this article. 
Wuhan. They are designed to be less sensitive to biases and confounding factors in the data and model assumptions. Without directly using case confirmation data in Wuhan, we avoid the potential biases in reporting and case confirmation in Wuhan, whereas because of the high level of domestic travel before the Lunar New Year in China, inference based on these data minimizes uncertainties and risk for potential misspecifications and biases in data and model assumptions.

\section{Methods}

\section{Methodologic Overview}

We developed 2 modeling approaches to infer the growth rate of the outbreak in Wuhan from data from provinces other than Hubei. In the first model, the first arrival model, we computed the likelihood of the arrival times of the first known cases in provinces outside of Hubei as a function of the exponential growing population of infected persons in Wuhan before late January. This calculation involved using domestic travel data to compute the probability that an infected person traveled from Wuhan to a given province as a function of the unknown actual number of infected persons in Wuhan and the probability that they traveled. The timings of the arrivals of the first infected persons in different provinces would reflect the rate of the epidemic growth in Wuhan.

In the second model, the case count model, we accounted for the detection of additional persons who were infected in Wuhan and received their diagnoses in other provinces and explicitly modeled those persons by using a hybrid deterministic-stochastic SEIR (susceptible-exposed-infectious-recovered) model. We then fitted this model to new daily case count data reported outside Hubei Province during the period before substantial transmission occurred outside of the province.

By using data collected outside Hubei Province, we minimized the effect of changes in surveillance intensity. By the time cases were confirmed in provinces outside Hubei, all of the provinces of China had access to diagnostic kits and were engaging in active surveillance of travelers out of Wuhan (e.g., using temperatures detectors and digital data to identify infected persons [9]) as the outbreak unfolded. Furthermore, the healthcare systems outside Hubei were not yet overwhelmed with cases and were actively searching for the first positive case, leading to much lower bias in the reporting in each province compared with the time series of confirmed cases in Wuhan.
Data

\section{Individual Case Reports}

We collected publicly available reports of 140 confirmed COVID-19 cases (mostly outside Hubei Province). These reports were published by the Chinese Centers for Disease Control and Prevention (China CDC) and provincial health commissions; accession dates were January 15-30, 2020 (Appendix 1 Table 1, https://wwwnc.cdc.gov/EID/article/26/7/200282-App1.xlsx). Many of the individual reports were also published on the China CDC official website (http://www.chinacdc.cn/jkzt/crb/zl/ szkb_11803) and the English version of the China CDC weekly bulletin (http://weekly.chinacdc.cn/ news/TrackingtheEpidemic.htm). These reports include demographic information as well as epidemiologic information, including potential periods of infection, and dates of symptom onset, hospitalization, and case confirmation. Most of the health commissions in provinces and special municipalities documented and published detailed information of the first or the first few patients with confirmed COVID-19. As a result, a unique feature of this dataset includes case reports of many of the first or the first few persons who were confirmed to have SARSCoV-2 virus infection in each province, where dates of departure from Wuhan were available.

\section{Travel Data}

We used the Baidu Migration server (https:/ / qianxi. baidu.com) to estimate the number of daily travelers in and out of Wuhan (Appendix 1 Table 2).

The server is an online platform summarizing mobile phone travel data hosted by Baidu Huiyan (https://huiyan.baidu.com).

\section{Calculations of $\mathbf{R}_{0}$ and Effect of Intervention Strategies}

We considered realistic distributions for the latent and infectious periods to calculate $R_{0}$. We described the methods we used to calculate $R_{0}$ and the effect of intervention strategies on the outbreak (Appendix 2, https:/ / wwwnc.cdc.gov/EID/article/26/7/20-0282App2.pdf).

\section{Results}

\section{Estimating Distributions of Epidemiologic Parameters}

We first translated reports from documents or news reports published daily from the China CDC website and official websites of health commissions across provinces and special municipalities in China during January 15-30, 2020. Altogether, we collected 
137 individual case reports from China and 3 additional case reports from outside of China (Appendix 1 Table 1).

By using this dataset, we estimated the basic parameter distributions of durations from initial exposure to symptom onset to hospitalization to discharge or death. Our estimate of the time from initial exposure to symptom onset (i.e., the incubation period) is 4.2 days (95\% CI 3.5-5.1 days) (Figure 1, panel A), based on 24 case reports. This estimated duration is generally consistent with a recent report by Guan et al. (10) showing that the median incubation period is 4 days. Our estimate is $\approx 1$ day shorter than 2 previous estimates $(1,11)$. One potential caveat of our estimation is that because most of the case reports we collected were from the first few persons detected in each province, this estimation might be biased toward patients with more severe symptoms if they are more likely to be detected.

The time from symptom onset to hospitalization showed evidence of time dependence (Figure 1, panel B; Appendix 2 Figure 1). Before January 18, the time from symptom onset to hospitalization was 5.5 days (95\% CI 4.6-6.6 days), whereas after January 18, the duration shortened significantly to 1.5 days $(95 \%$ CI $1.2-1.9$ days) ( $p<0.001$ by Mann-Whitney $U$ test). The change in the distribution coincides with news reports of potential human-to-human transmission and upgrading of emergency response level to Level 1 by the China CDC. The emerging consensus about the risk for COVID-19 probably led to substantial behavior changes among symptomatic persons, in terms of seeking more timely medical care during this period. However, because most of the individual reports were collected in provinces other than Hubei, the
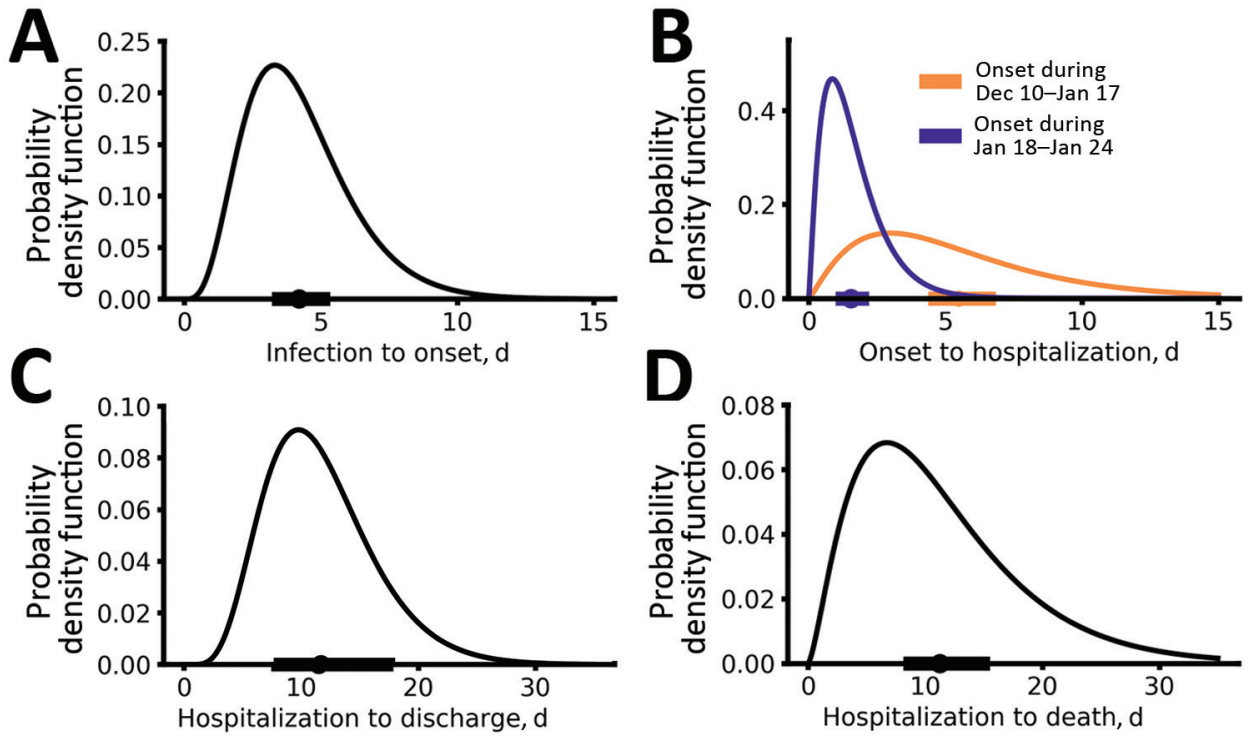

change in durations might only reflect changes in the rest of China (rather than in Hubei). We also found that the time from initial hospital admittance to discharge was 11.5 days (95\% CI 8.0-17.3 days) (Figure 1, panel C) and from initial hospital admittance to death was 11.2 days (95\% CI 8.7-14.9 days) (Figure 1, panel D). The time from symptom onset to death was estimated to be 16.1 days (95\% CI 13.1-20.2 days).

\section{Estimating the Growth Rate of the Outbreak in Wuhan in January 2020}

Moving from empirical estimates of basic epidemiologic parameters to an understanding of the early growth rates of COVID-19 requires model-based inference and data. We first collected real-time travel data during the epidemic by using the Baidu Migration server, which provides real-time travel patterns in China based on mobile-phone positioning services (Figure 2, panel A; Appendix 1 Methods, Table 2). We estimated that, before the January 23 lockdown of the city, $\approx 40,000-140,000$ people in Wuhan traveled to destinations outside Hubei Province each day (Figure 2, panel B). The extensive travel before the Lunar New Year was probably an important driver of the spread of COVID-19 in China.

We then integrated spatiotemporal domestic travel data to infer the outbreak dynamics in Wuhan by using two mathematical approaches (Appendix 2; conceptual framework depicted in Figure 3, panel A). The first-arrival model uses a unique feature of our case report dataset whereby the dates of departure from Wuhan for many of the first persons who were confirmed with SARS-CoV-2 infection in each province were known (Appendix 1 Table 1). We assumed an exponential growth for the total infected
Figure 1. Epidemiologic characteristics of early dynamics of coronavirus disease outbreak in China. Distributions of key epidemiologic parameters: durations from infection to symptom onset $(A)$, from symptom onset to hospitalization (B), from hospitalization to discharge (C), and from hospitalization to death (D). Filled circles and bars on $x$-axes denote the estimated means and $95 \% \mathrm{Cls}$. 


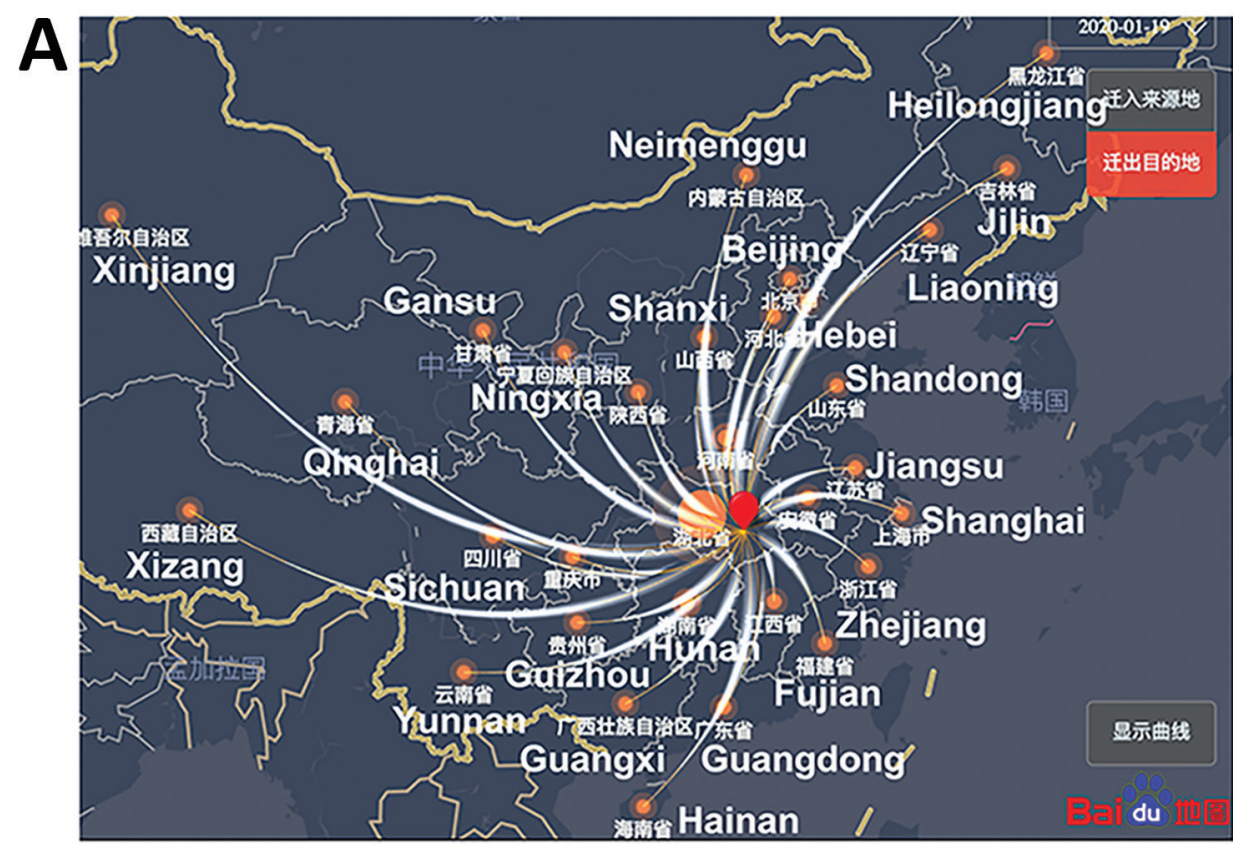

Figure 2. Extremely high level of travel from Wuhan, Hubei Province, to other provinces during January 2020 , as estimated by using high-resolution and real-time travel data, China. A) A modified snapshot of the Baidu Migration online server interface showing the human migration pattern out of Wuhan (red dot) on January 19, 2020. Thickness of curved white lines denotes the size of the traveler population to each province. The names of most of the provinces are shown in white. B) Estimated daily population sizes of travelers from Wuhan to other provinces.
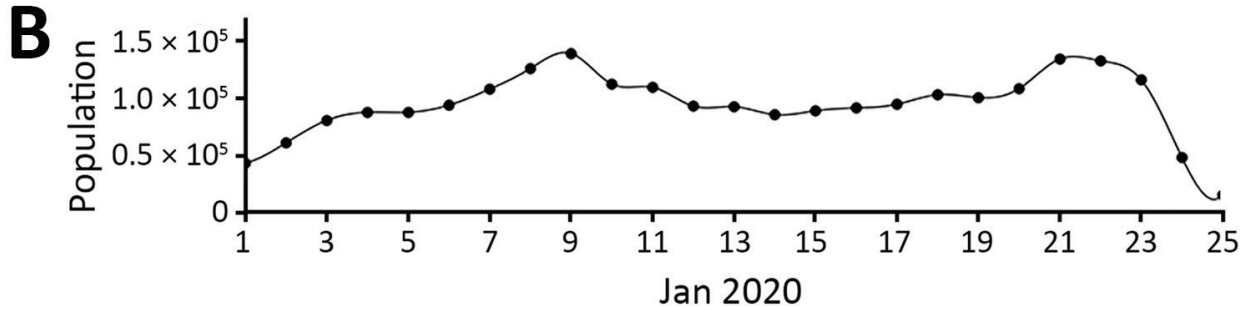

population $I^{*}$ in Wuhan, $I^{*}(t)=\mathrm{e}^{\mathrm{r}\left(t-t_{0}\right)}$, where $I^{*}$ includes infected persons who are asymptomatic or symptomatic, $r$ is the exponential growth rate, and $t_{0}$ is the theoretical time of the exponential growth initiation, so that $I^{*}\left(t_{0}\right)=1$ in the deterministic model. We call $t_{0}$ a "theoretical" time in the sense that it should not be interpreted as the time of first infection in a population. We should expect that $t_{0}$ is later than the date of the first infection because multiple spillover events from the animal reservoir might be needed to establish sustained transmission and stochasticity might play a large role in initial dynamics before the onset of exponential growth (12-14).

We used travel data for each of the provinces (Appendix 1 Table 3 ) and the earliest times that an infected person arrived in a province, across a total of 26 provinces (Figure 3, panel B), to infer $r$ and $t_{0}$ (Appendix 2). Model predictions of arrival times in the 26 provinces fitted the actual data well (Appendix 2 Figure 2). The growth rate $r$ is estimated to be 0.29 /day (95\% CI 0.21 0.37 / day), corresponding to a doubling time of 2.4 days (95\% CI 1.9-3.3 days). $t_{0}$ is estimated to be December 20, 2019 (95\% CI December 11-26). As we show later, there exist larger uncertainties in the estimation of $t_{0}$.
We further estimated that the total infected population size in Wuhan was $\approx 4,100$ (95\% CI 2,4236,178 ) on January 18 (Appendix 2 Figure 3), which is consistent with a recently posted estimate (7). The estimated number of infected persons was $\approx 18,700$ $(95 \%$ CI 7,147-38,663) on January 23 (i.e., the date when Wuhan started its lockdown). We projected that without any control measures, the infected population would be $\approx 233,400$ (95\% CI 38,757-778,278) by the end of January.

An alternative model, the case count approach, used daily new case counts of persons who had COVID-19 diagnosed in other provinces but who had been in Hubei Province within 14 days of becoming symptomatic. This model uses data beyond the first appearance of an infected person from Wuhan but also accounts for the stochastic nature of the process by using a hybrid model. In this model, the infected population in Wuhan was described with a deterministic model, whereas the infected persons who traveled from Wuhan to other provinces were tracked with a stochastic SEIR (susceptible-exposed-infectious-recovered) model (12). We restricted the data to the period of January 

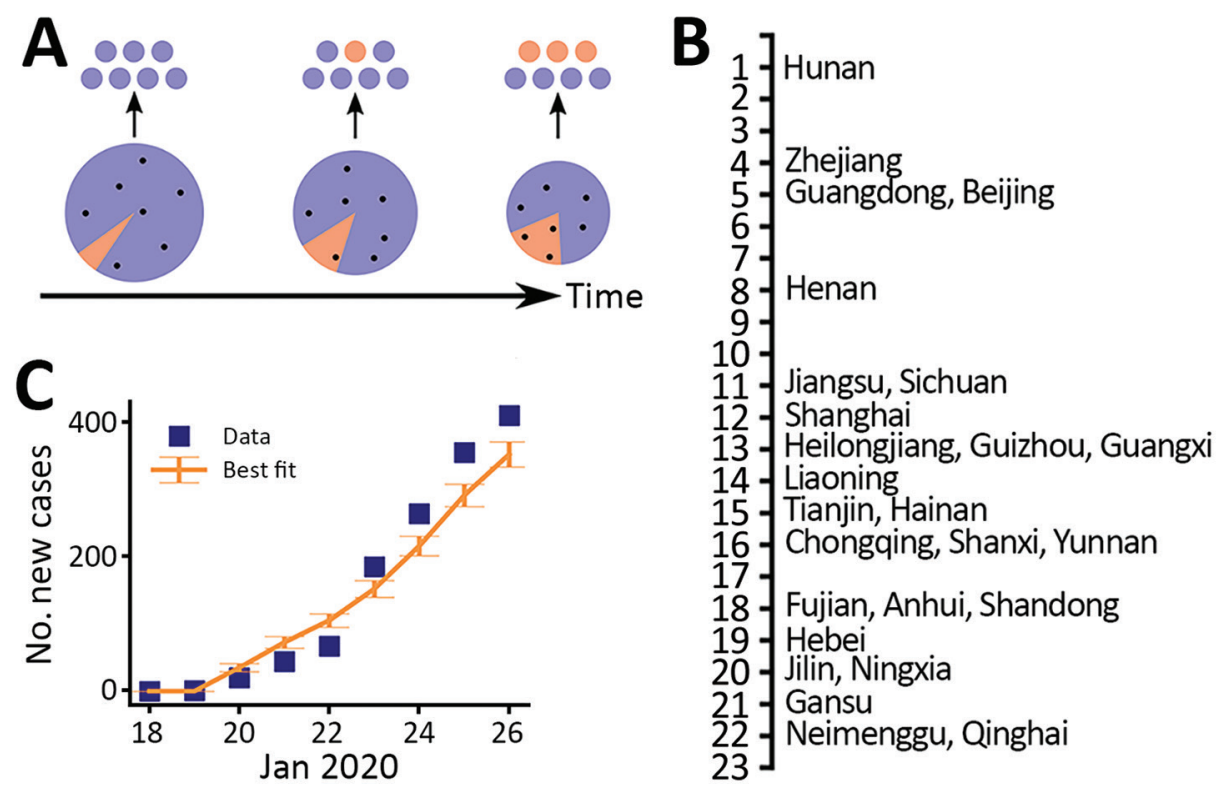

Figure 3. Estimates of the exponential growth rate and the date of exponential growth initiation of the coronavirus disease outbreak in China based on 2 different approaches. A) Schematic illustrating the export of infected persons from Wuhan. Travelers (dots) are assumed to be random samples from the total population (whole pie). Because of the growth of the infected population (orange pie) and the shrinking size of the total population in Wuhan over time, probability of infected persons traveling to other provinces increases (orange dots). B) The dates of documented first arrivals of infected persons in 26 provinces. C) Best fit of the case count model to daily counts of new cases (including only imported cases) in provinces other than Hubei. Error bars indicate SDs.

19-26, when new cases reported were mostly infections imported from Wuhan (i.e., indicative of the dynamics in Wuhan). The transitions of the infected persons from symptom onset to hospitalization and then to case confirmation were assumed to follow the distributions inferred from the case report data (Appendix 2). Simulation of the model using best-fit parameters showed that the model described the observed case counts over time well (Figure 3, panel C). The estimated theoretical time $\left(t_{0}\right)$ is December 16, 2019 (95\% CI December 12-21), and the exponential growth rate is $0.30 /$ day $(95 \%$ CI 0.26-0.34/day). These estimates are consistent with estimates in the first arrival approach (Figure 4; Appendix 2 Figure 4).

In both models, we assumed perfect detection (i.e., of infected cases outside of Hubei Province). However, a certain fraction of cases probably was not reported. To investigate the robustness of our estimates, we performed extensive sensitivity analyses to test 23 different scenarios of surveillance intensity (Appendix 2). First, we tested the assumption that a constant fraction of infected persons (e.g., persons with mild or no symptoms) (15) were not detected. We found that under this assumption, $t_{0}$ would be earlier than our estimate but the estimation of the growth rate remained the same (Appendix 1 Table 4). Second, we tested the assumption that the intensity of surveillance increases over the period of data collection, although this scenario is less likely because of the intensive surveillance implemented outside Hubei Province. We found that our data in general do not support this hypothesis on the basis of corrected Akaike Information criterion scores (Appendix 1 Table 4). However, if the intensity of surveillance outside Hubei Province increased over the period of January, we would predict a lower growth rate than the estimate we just described. For the worst-case scenario considered, we estimated the growth rate of the outbreak to be 0.21 /day (Appendix 2).

\section{Other Evidence of a High Growth Rate of the Outbreak in Wuhan}

In addition to using 2 modeling approaches, we looked for other evidence of a high outbreak growth rate to cross-validate our estimations. We found that the time series of reported deaths in Hubei, which is less subject to the biases of the confirmed case counts, is simply not consistent with a growth rate of 0.1 / day (Appendix 2 Figure 5). As the infected population grows, the number of death cases will grow at the same rate but with a delayed onset corresponding to the time from infection to death. Fitting a simple exponential growth model to the number of reported deaths in Hubei during late January 2020 yields an estimate of $0.22-0.27 /$ day, which is within the $95 \%$ CI of the estimation we previously described.

Overall, these analyses suggest that although there exist uncertainties depending on the level of surveillance, the exponential growth rate of the outbreak is probably $0.21-0.3 /$ day. This estimation is much higher than previous reports, in which the growth rate was estimated to be $0.1-0.14$ / day $(1,3-5)$. 


\section{Estimating $\mathbf{R}_{0}$}

The basic reproductive number, $R_{0^{\prime}}$ is dependent on the exponential growth rate of an outbreak, as well as additional factors such as the latent period (the time from infection to infectiousness) and the infectious period $(16,17)$, both of which cannot be estimated directly from the data. Following the approach by Wearing and Rohani (16), we found that with a high growth rate of the outbreak, $R_{0}$ is in general high and the longer the latent and the infectious periods, the higher the estimated $R_{0}$ (Appendix 2 Figure 6).

To derive realistic values of $R_{0}$, we used previous estimates of serial intervals for COVID-19. The serial interval is estimated to be $\approx 7-8$ days based on data collected early in the outbreak in Wuhan (1). More recent data collected in Shenzhen Province, China, suggests that the serial interval is dependent on the time to hospital isolation (Q. Bi et al., unpub. data, https:/ / doi.org/10.1101/2020.03.03.20028423). When infected persons are isolated after 5 days of symptoms (a probable scenario for the early outbreak in Wuhan, where the public was not aware of the virus and few interventions were implemented), the serial interval is estimated to be 8 days (Q. Bi et al., unpub. data). Thus, these results suggest a serial interval of 7-8 days. With this serial interval, we sampled latent and infectious periods within wide biologically plausible ranges (Appendix 2) and estimated the median $R_{0}$ to be 5.8 (95\% CI 4.4-7.7) (Figure 5, panel A). To include a wider range of serial interval (i.e., 6-9 days) (Figure 5, panel A; Appendix 2 Figure 6), given the uncertainties in these estimations, we estimated that the median of estimated $\mathrm{R}_{0}$ is 5.7 (95\% CI of 3.8-8.9) (Figure 5 , panel B). The estimated $R_{0}$ can be lower if the serial interval is shorter. However, recent studies reported that persons can be infectious for a long period, such as 1-3 weeks after symptom onset $(18 ; \mathrm{R}$. Woelfel

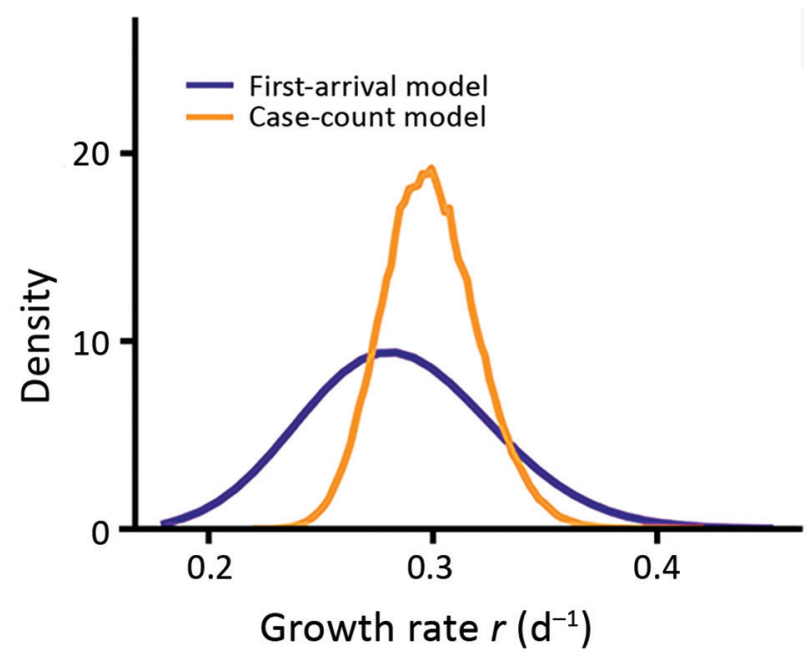

Figure 4. Marginalized likelihoods of growth rate $(r)$ for 2 inference approaches to estimates the exponential growth rate of the coronavirus disease outbreak in China.

et al., unpub data. https://doi.org/10.1101/2020.03. 05.20030502); thus, we believe that a mean serial interval shorter than 6 days is unlikely during the early outbreak in Wuhan, where infected persons were not rapidly hospitalized.

\section{Implications for Intervention Strategies}

The $R_{0}$ values we estimated have important implications for predicting the effects of pharmaceutical and nonpharmaceutical interventions. For example, the threshold for combined vaccine efficacy and herd immunity needed for disease extinction is calculated as $1-1 / R_{0}$. At $R_{0}=2.2$, this threshold is only $55 \%$. But at $R_{0}=5.7$, this threshold rises to $82 \%$ (i.e., $>82 \%$ of the population has to be immune, through either vaccination or prior infection, to achieve herd immunity to stop transmission).
Figure 5. Estimation of the basic reproductive number $\left(R_{0}\right)$, derived by integrating uncertainties in parameter values, during the coronavirus disease outbreak in China. A) Changes in $R_{0}$ based on different growth rates and serial intervals. Each dot represents a calculation with mean latent period (range 2.2-6 days) and mean infectious periods (range $4-14$ days). Only those estimates falling within the range of serial intervals of interests were plotted. B) Histogram summarizing the estimated $R_{0}$ of all dots in panel A (i.e., serial interval ranges of $6-9$ days). The median $\mathrm{R}_{0}$ is $5.7(95 \% \mathrm{Cl} 3.8-8.9)$.
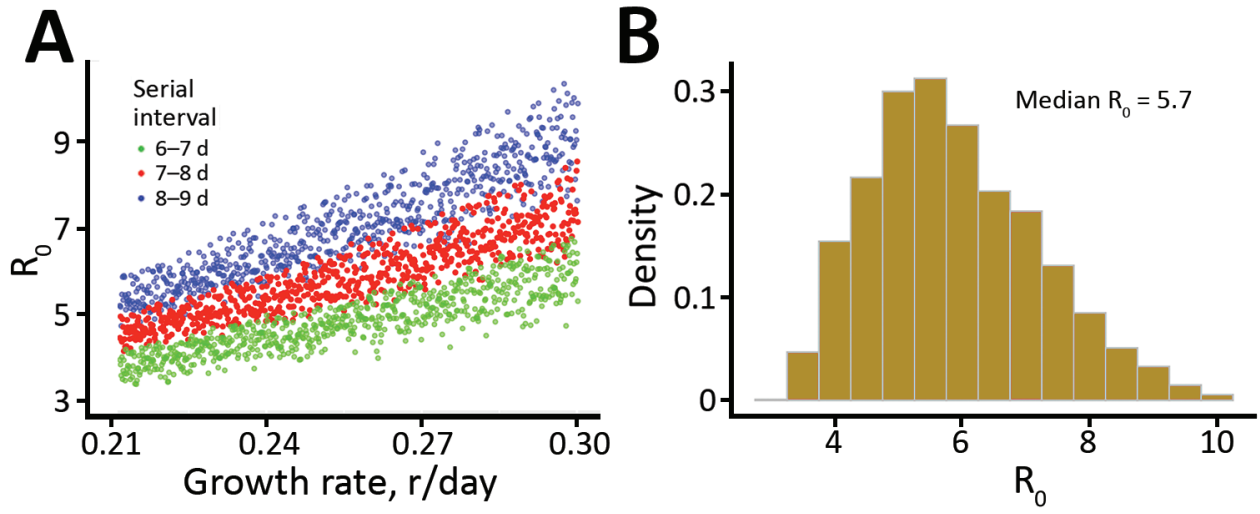


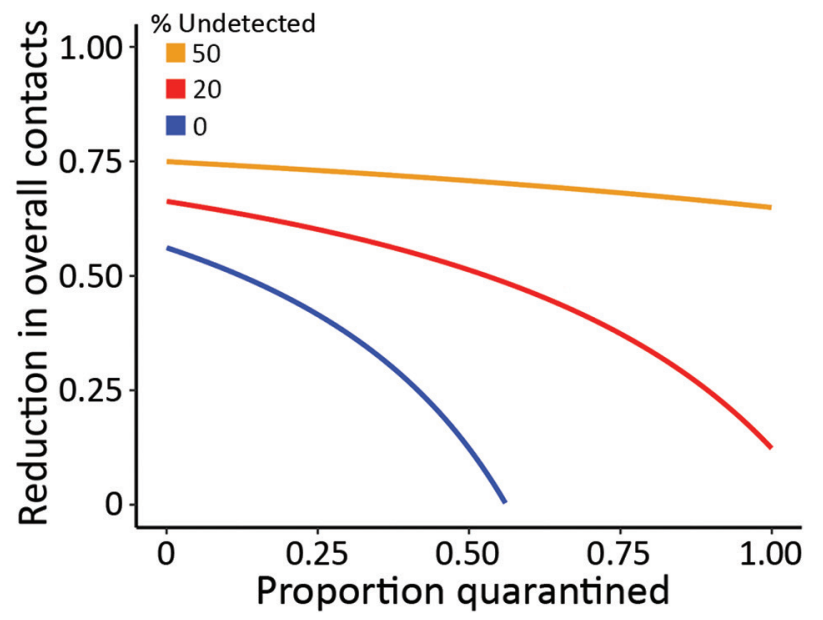

Figure 6. Levels of minimum efforts of intervention strategies needed to control the spread of severe acute respiratory syndrome coronavirus 2 , (i.e. reducing the reproductive number to $<1$ ), during the coronavirus disease outbreak in China. Strategies considered were quarantine of infected persons and persons who had contact with them ( $x$-axis) and population-level efforts to reduce overall contact rates ( $y$-axis). Percentages denote the percentages of transmissions driven by infected persons that were not detected by surveillance as a result of asymptomatic infection, mild-to-moderate illness or low surveillance intensity.

We then evaluated the effectiveness for nonpharmaceutical interventions, such as contact tracing, quarantine, and social distancing, by using the framework by Lipsitch et al. (19) (Appendix 2). We extended the framework to consider a fraction of transmission occurring from infected persons who would not be identified by surveillance and can transmit effectively (15). This fraction is determined by the fraction of actual asymptomatic persons and the extent of surveillance efforts to identify these persons and persons with mild-to-moderate symptoms. Results show that quarantine and contact tracing of symptomatic persons can be effective when the fraction of unidentified persons is low. However, when $20 \%$ of transmission is driven by unidentified infected persons, high levels of social distancing efforts will be needed to contain the virus (Figure 6), highlighting the importance of early and effective surveillance, contact tracing, and quarantine. Future field, laboratory, and modeling studies aimed to address the unknowns, such as the fraction of asymptomatic persons, the extent of their transmissibility depending on symptom severity, the time when persons become infectious, and the existence of superspreaders are needed to accurately predict the impact of various control strategies (20).

\section{Discussion}

In this study, we estimated several basic epidemiologic parameters, including the incubation period (4.2 days), a time dependent duration from symptom onset to hospitalization (changing from 5.5 days in early January to 1.5 days in late January outside Hubei Province), and the time from symptom onset to death (16.1 days). By using 2 distinct approaches, we estimated the growth rate of the early outbreak in $\mathrm{Wu}$ han to be $0.21-0.30$ per day (a doubling time of 2.3-3.3 days), suggesting a much faster rate of spread than initially measured. This finding would have important implications for forecasting epidemic trajectories and the effect on healthcare systems as well as for evaluating the effectiveness of intervention strategies.

We found $R_{0}$ is likely to be 5.7 given our current state of knowledge, with a broad 95\% CI (3.8-8.9). Among many factors, the lack of awareness of this new pathogen and the Lunar New Year travel and gathering in early and mid-January 2020 might or might not play a role in the high $R_{0}$. A recent study based on structural analysis of the virus particles suggests SARS-CoV-2 has a much higher affinity to the receptor needed for cell entry than the 2003 SARS virus (21), providing a molecular basis for the high infectiousness of SARS-CoV-2.

How contagious SARS-CoV-2 is in other countries remains to be seen. Given the rapid rate of spread as seen in current outbreaks in Europe, we need to be aware of the difficulty of controlling SARS$\mathrm{CoV}-2$ once it establishes sustained human-to-human transmission in a new population (20). Our results suggest that a combination of control measures, including early and active surveillance, quarantine, and especially strong social distancing efforts, are needed to slow down or stop the spread of the virus. If these measures are not implemented early and strongly, the virus has the potential to spread rapidly and infect a large fraction of the population, overwhelming healthcare systems. Fortunately, the decline in newly confirmed cases in China and South Korea in March 2020 and the stably low incidences in Taiwan, Hong Kong, and Singapore strongly suggest that the spread of the virus can be contained with early and appropriate measures.

\section{Acknowledgments}

We thank Alan Perelson, Christiaan van Dorp, and Ruy Ribeiro for suggestions and critical reading of the manuscript and Weili Yin for help with collecting and translating documents from provincial health commission websites.

S.S. and R.K. received funding from the Defense Advanced Research Projects Agency (grant no. HR0011938513) and the Laboratory Directed Research and Development Rapid 
Response Program through the Center for Nonlinear Studies at Los Alamos National Laboratory. C.X. received funding from Laboratory Directed Research and Development Program. E.R.S. received funding from the National Institutes of Health (grant no. R01AI135946). The funders had no role in study design, data collection and analysis, decision to publish, or preparation of the manuscript.

Author contributions: R.K. and N.H. conceived the project; R.K. collected data; S.S., Y.T.L., C.X., and R.K. performed analyses; S.S., Y.T.L., E.R.S., N.H., and R.K. wrote and edited the manuscript.

Authors declare no competing interests. All data are available in the main text and in Appendices 1 and 2.

\section{About the Author}

Dr. Sanche is a postdoctoral research associate at Los Alamos National Laboratory, Los Alamos, New Mexico, USA. His primary research interest lies in complex disease dynamics inferred from data science and mathematical modeling. Dr. Lin is also a postdoctoral research associate at Los Alamos National Laboratory. His primary research interest lies in applied stochastic processes, biological physics, statistical inference, and computational system biology.

\section{References}

1. Li Q, Guan X, Wu P, Wang X, Zhou L, Tong Y, et al. Early transmission dynamics in Wuhan, China, of novel coronavirus-infected pneumonia. N Engl J Med. 2020;382:1199-207. https://doi.org/10.1056/NEJMoa2001316

2. WHO. Pneumonia of unknown cause-China [cited 2020 Jan 30]. https://www.who.int/csr/don/05-january2020-pneumonia-of-unkown-cause-china

3. Wu JT, Leung K, Leung GM. Nowcasting and forecasting the potential domestic and international spread of the 2019-nCoV outbreak originating in Wuhan, China: a modelling study. Lancet. 2020;395:689-97. https:// doi. org/10.1016/S0140-6736(20)30260-9

4. Riou J, Althaus CL. Pattern of early human-to-human transmission of Wuhan 2019 novel coronavirus (2019-nCoV), December 2019 to January 2020. Euro Surveill. 2020;25:25. https://doi.org/10.2807/1560-7917.ES.2020.25.4.2000058

5. Du Z, Wang L, Cauchemez S, Xu X, Wang X, Cowling BJ, et al. Risk for transportation of coronavirus disease from Wuhan to other cities in China. Emerg Infect Dis. 2020;26:1049-52. https:// doi.org/10.3201/eid2605.200146

6. Novel Coronavirus Pneumonia Emergency Response Epidemiology Team. The epidemiological characteristics of an outbreak of 2019 novel coronavirus diseases (COVID-19) in China [in Chinese]. Zhonghua Liu Xing Bing Xue Za Zhi. 2020;41:145-51.

7. Imai N, Dorigatti I, Cori A, Donnelly C, Riley S. Ferguson. Report 2: estimating the potential total number of novel coronavirus cases in Wuhan City, China [cited 2020 Feb 2]. https://www.imperial.ac.uk/media/imperial-college/
medicine/sph/ide/gida-fellowships/Imperial-CollegeCOVID19-update-epidemic-size-22-01-2020.pdf

8. Imai N, Dorigatti I, Cori A, Riley S, Ferguson NM. Estimating the potential total number of novel coronavirus cases in Wuhan City, China [cited 2020 Feb 2]. https:/ / www. imperial.ac.uk/media/imperial-college/medicine/sph/ide/ gida-fellowships/2019-nCoV-outbreak-report-17-01-2020.pdf

9. Hongyang L. Railway corporation using big data to trace potential virus carrier. ChinaDailyNews [cited 2020 Feb 1]. https:/ / www.chinadaily.com.cn/a/202001/30/ WS5e329ca2a310128217273b89.html

10. Guan WJ, Ni ZY, Hu Y, Liang WH, Ou CQ, He JX, et al.; China Medical Treatment Expert Group for Covid-19. Clinical characteristics of coronavirus disease 2019 in China. N Engl J Med. 2020 Feb 28 [Epub ahead of print]. https:/ / doi.org/10.1056/NEJMoa2002032

11. Lauer SA, Grantz KH, Bi Q, Jones FK, Zheng Q, Meredith HR, et al. The incubation period of coronavirus disease 2019 (COVID-19) from publicly reported confirmed cases: estimation and application. Ann Intern Med. 2020 Mar 10 [Epub ahead of print]. https://doi.org/10.7326/M20-0504

12. Anderson RM, May RM. Infectious diseases of humans: dynamics and control. Oxford Science Publications. Oxford: Oxford University Press; 1991. p. 768.

13. Lloyd-Smith JO, Schreiber SJ, Kopp PE, Getz WM. Superspreading and the effect of individual variation on disease emergence. Nature. 2005;438:355-9. https:/ / doi.org/ 10.1038 /nature 04153

14. Romero-Severson EO, Ribeiro RM, Castro M. Noise is not error: detecting parametric heterogeneity between epidemiologic time series. Front Microbiol. 2018;9:1529. https:/ / doi.org/10.3389/fmicb.2018.01529

15. Rothe C, Schunk M, Sothmann P, Bretzel G, Froeschl G, Wallrauch C, et al. Transmission of 2019-nCoV infection from an asymptomatic contact in Germany. N Engl J Med. 2020;382:970-1. https://doi.org/10.1056/NEJMc2001468

16. Wearing HJ, Rohani P, Keeling MJ. Appropriate models for the management of infectious diseases. PLoS Med. 2005;2:e174. https:// doi.org/10.1371/journal.pmed.0020174

17. Lloyd AL. The dependence of viral parameter estimates on the assumed viral life cycle: limitations of studies of viral load data. Proc Biol Sci. 2001;268:847-54. https:/ / doi.org/10.1098/rspb.2000.1572

18. Zhou F, Yu T, Du R, Fan G, Liu Y, Liu Z, et al. Clinical course and risk factors for mortality of adult inpatients with COVID-19 in Wuhan, China: a retrospective cohort study. Lancet. 2020;395:1054-62. https:/ / doi.org/10.1016/ S0140-6736(20)30566-3

19. Lipsitch M, Cohen T, Cooper B, Robins JM, Ma S, James L, et al. Transmission dynamics and control of severe acute respiratory syndrome. Science. 2003;300:1966-70. https://doi.org/10.1126/science.1086616

20. Fraser C, Riley S, Anderson RM, Ferguson NM. Factors that make an infectious disease outbreak controllable. Proc Natl Acad Sci U S A. 2004;101:6146-51. https:/ / doi.org/10.1073/ pnas.0307506101

21. Wrapp D, Wang N, Corbett KS, Goldsmith JA, Hsieh CL, Abiona O, et al. Cryo-EM structure of the 2019-nCoV spike in the prefusion conformation. Science. 2020;367:1260-3. https:// doi.org/10.1126/science.abb2507

Address for correspondence: Ruian Ke, T-6 Theoretical Biology and Biophysics, Mailstop K710, Los Alamos National Laboratory, NM 87544, USA; email: rke@lanl.gov 